\title{
Simulation of input electron noise in the free-electron laser
}

\author{
C. Penman \\ Faculteit der Technische Natuurkunde, Universiteit Twente, Postbus 217, 7500 AE Enschede, Netherlands
}

and

B.W.J. McNeil

AEA Technology, Culham Laboratory, Culham OX14 3DB, UK

Received 2 January 1992

\begin{abstract}
We present a calculation of the shot noise to be used as initial condition for the electron-beam phase-variables in numerical simulations of the free-electron laser.
\end{abstract}

Numerical integration of the free-electron laser equations usually starts with a small arbitrary value of input field, to allow the FEL instability to show itself. This procedure fails to reproduce the start-up of the real laser and the time or distance to saturation will depend on the arbitrary initial value. The procedure is obviously valid only for initial field-values larger than the random fluctuations in the real system; even if the level of these fluctuations is used as an initial value, the time to saturation for start-up from noise will be incorrectly predicted, since the initial evolution of the electron bunching and field is an incoherent process with linear growth, which is superseded only gradually by a coherent process with exponential growth. The purpose of the present communication is to explain how noise in the electron beam should be simulated on a computer.

The FEL equations consist of a Maxwell wave equation for the radiation field envelope $a$ coupled with a number of Lorentz equations for the ponderomotive phases $\theta_{j}$ and energies of the electron. In the Compton limit, for example, using the normalization of ref. [1] and the coordinate $\tau$ to represent the electron-beam local time, the equations are

$\partial^{2} \theta_{j} / \partial Z^{2}=-\left[a \exp \left(i \theta_{j}\right)+\right.$ c.c. $]$, $\left(\frac{\partial}{\partial Z}-\frac{1}{V} \frac{\partial}{\partial \tau}\right) a=\langle\exp (-\mathrm{i} \theta)\rangle$

The rate of slip or relative movement between light and electrons is given by $V \equiv \beta_{z} /\left(1-\beta_{z}\right)$. In the absence of an initial field the detuning has been taken to be zero. To obtain correct results from a numerical integration of these equations, a statistical analysis of the noise in the electron phase-distribution is needed, so that the effect of noise on the average denoted by angle brackets on the right side of eq. (2) can be corrected for the unrealistically small number of electron phase-values necessarily used on a computer. The method presented here, based on a comparison of statistics in the real and the numerical situations, has been compared with the predictions of low-gain start-up theory [2].

The simplest way of obtaining a realistic simulation of the noise in the input electron distribution with a limited number of electrons is to have the noise entirely in the electrons' positions, assuming zero variance in momentum, and to reproduce the first and second moments of the relevant quantity for the real distribution of positions; namely the mean and variance, taken over the several realizations in an ensemble, of the initial value of 
$\langle\exp (-\mathrm{i} \theta)\rangle=\frac{1}{N} \sum_{j=1}^{N} \exp \left(-\mathrm{i} \theta_{j}\right)$.

(The present description applies to the case where $\langle\exp (-\mathrm{i} \theta)\rangle$ is actually evaluated as an average by the computer program; the method can be extended to the more efficient procedure in which the average is replaced by an integral with respect to initial phase, performed by higher-order quadrature methods. ) The problem, therefore, is to reproduce the mean $E(\langle\exp (-\mathrm{i} \theta)\rangle)$ and variance $V(\langle\exp (-\mathrm{i} \theta)\rangle)$ of the quantity in eq. (3) using, instead of the real distribution and number $N$ of electrons an artificial distribution with a much smaller number $n$. The method will be described for the real part of the quantity; the results are the same for the imaginary part.

The standard deviation of the actual distribution is found by considering the distribution of emission times, or times of passing a fixed point at the beginning of the magnet. This distribution is uniform, and the number $m$ of electrons which pass the fixed point in time $T$ has a Poisson distribution. For a sufficient value of $T$ the standard deviation $[V(m(T))]^{1 / 2}$ of the number that pass will be negligible compared with the mean $\bar{m}(T)$; for a Poisson distribution the standard deviation is $\bar{m}^{1 / 2}$ so that any time for which $\bar{m} \gg 1$ is sufficient. Practical values of the current justify the choice of the optical period $\lambda / c$ for $T$. The variance (over realizations) of $\cos \theta_{j}$ (for the $j$ th electron) is then

$$
\begin{aligned}
& V\left(\cos \theta_{j}\right)=E\left(\cos ^{2} \theta_{j}\right)-E\left(\cos \theta_{j}\right)^{2} \\
& =\frac{1}{2 \pi} \int_{0}^{2 \pi} \cos ^{2} \theta_{j} \mathrm{~d} \theta_{j}-\left(\frac{1}{2 \pi} \int_{0}^{2 \pi} \cos \theta_{j} \mathrm{~d} \theta_{j}\right)^{2}=\frac{1}{2},
\end{aligned}
$$

since the mean is 0 .

The values of $\cos \theta_{i}$ and $\cos \theta_{j}$ for different electrons $(i \neq j)$ are uncorrelated in the ensemble; that is, the covariance satisfies

$E\left(\cos \theta_{i} \cos \theta_{j}\right)=0, \quad(i \neq j)$.

Consequently

$E\left(\sum_{i, j} \cos \theta_{i} \cos \theta_{j}\right)=E\left(\sum_{i, j} \cos ^{2} \theta_{j}\right)=\sum_{j} E\left(\cos ^{2} \theta_{j}\right)$,

and the variance of $\langle\cos \theta\rangle$ is therefore

$$
\begin{gathered}
V(\langle\cos \theta\rangle)=V\left(\frac{1}{N} \sum \cos \theta_{j}\right) \\
=\frac{1}{N^{2}} \sum V\left(\cos \theta_{j}\right)=\frac{1}{2 N} .
\end{gathered}
$$

Pseudo-random numbers uniformly distributed within an interval are available on every computer. The artificial distribution devised therefore consists of uniformly-distributed departures from even spacing up to some maximum. The maximum permitted departure needed to reproduce the real standard deviation can be calculated from the number of artificial electrons. If the maximum departure is $\delta$ then the mean and mean square over realizations, for the $j$ th electron, are

$$
\begin{aligned}
& E\left(\cos \theta_{j}\right)=\frac{1}{2 \delta} \int_{2 \pi j / n-\delta}^{2 \pi j / n+\delta} \cos \theta_{j} \mathrm{~d} \theta_{j} \\
& =\cos (2 \pi j / n) \operatorname{sinc}(\delta), \quad(\operatorname{sinc}(x) \equiv \sin (x) / x)
\end{aligned}
$$

and

$E\left(\cos ^{2} \theta_{j}\right)=\frac{1}{2}[1+\cos (2 \pi j / n) \operatorname{sinc}(2 \delta)]$.

These give a variance

$$
\begin{aligned}
& V\left(\cos \theta_{j}\right)=\frac{1}{2}\left\{1-\operatorname{sinc}^{2}(\delta)\right. \\
& \left.\quad+\cos (4 \pi j / n)\left[\operatorname{sinc}(2 \delta)-\operatorname{sinc}^{2}(\delta)\right]\right\},
\end{aligned}
$$

for the $j$ th electron. The mean and variance of $\langle\cos \theta\rangle$ are therefore respectively 0 and

$$
\begin{aligned}
& V\left(\frac{1}{n} \sum \cos \theta_{j}\right)=\frac{1}{n^{2}} \sum V\left(\cos \theta_{j}\right) \\
& =\left(1-\sin c^{2} \delta\right) / 2 n .
\end{aligned}
$$

Equating eqs. (4) and (5) then gives

$\operatorname{sinc}^{2} \delta=1-n / N$,

or, for $n \ll N$,

$\delta^{2} \approx 3 n / N$.

Thus in a program the $j$ th electron phase-value should be initialized to

$\exp \{\mathrm{i}[(j / n) 2 \pi+2 r \delta]\}$,

where $\delta$ is given by eq. (7) and $r$ is a pseudo-random variable uniformly distributed between 0 and 1 . This 
procedure has been shown to give good agreement with theory in the low-gain regime [2], has recently been applied to the high-gain Compton regime [3], and will shortly be applied to the Raman regime [4].

\section{References}

[1] R. Bonifacio, F. Casagrande, G. Gerchioni, L. de Salvo Souza and P. Pierini: One-dimensional theory of a free-electron laser amplifier: steady state and superradiance, in: High gain, high power free-electron laser: physics and application to $\mathrm{TeV}$ particle acceleration, eds. R. Bonifacio, L. de Salvo Souza and C. Pellegrini (North-Holland, Amsterdam, 1989).
[2] J.N. Elgin and C. Penman, Phys. Lett. A 114 (1986) 227.

[3] C. Penman, Development of coherence in the high-gain Compton free-electron laser (submitted to Optics Comm.).

[4] C. Penman, Development of coherence in the Raman freeelectron laser (in preparation). 